\title{
Clinical Types and Outcome of Minor Ischemic Stroke in Northern China: A Retrospective Cohort Study
}

\author{
Guangsheng Wang ${ }^{*}$, Daoming Tong1 ${ }^{*}$, Xiaodong Chen ${ }^{1}$, Tonghui Yang1, Yeting Zhou ${ }^{2}$ \\ ${ }^{1}$ Department of Neurology, The Affiliated Shuyang People' Hospital, Xuzhou Medical University, Xuzhou, China \\ ${ }^{2}$ Medical Evaluation Center, The Affiliated Shuyang People' Hospital, Xuzhou Medical University, Xuzhou, China \\ Email: tongdaoing@163.com
}

How to cite this paper: Wang, G.S., Tong, D.M., Chen, X.D., Yang, T.H. and Zhou, Y.T. (2017) Clinical Types and Outcome of Minor Ischemic Stroke in Northern China: A Retrospective Cohort Study. World Journal of Neuroscience, 7, 95-105. https://doi.org/10.4236/wjns.2017.71009

Received: December 30, 2016

Accepted: February 6, 2017

Published: February 9, 2017

Copyright $(0) 2017$ by authors and Scientific Research Publishing Inc. This work is licensed under the Creative Commons Attribution International License (CC BY 4.0).

http://creativecommons.org/licenses/by/4.0/ (c) (i) Open Access

\begin{abstract}
Background: As a common and high incidence of disease, the minor ischemic stroke (MIS) has become an important public health problem. The aim of this study was to address whether patients with MIS have different types and outcome in the elderly in northern China. Methods: A retrospective cohort of consecutive patients was selected for study; all registered neurologic outpatients of the tertiary teaching hospital in northern Jiangsu, China between February, 2011 and February, 2012. A total of 433 outpatients, clinically only having had an initial visit and a MRI study of the brain, were enrolled. Results: Of 433 outpatients, 247 (57.0\%) patients with MIS were diagnosed. The clinical types of MIS included stable MIS in 58.3\%, acute progressive MIS in $5.3 \%$, and chronic progressive MIS in $36.4 \%$ of patients. After adjusted Odd ratio (OR), only aged (OR, 1.0; 95\% confidence interval [CI], $1.007-1.087, p$ $=0.021)$, episode duration (OR, $1.8 ; 95 \% \mathrm{CI}, 1.011-1.024, p=0.001)$, initial number of MIS (OR, 1.1; 95\% CI, $1.047-1.207, p=0.001$ ), and infarcts volume (OR, 1.8; 95\% CI, $1.253-2.681, p=0.002)$ were independently associated with stable MIS and progressive MIS. Total survival was favorable among groups $(p=0.094)$, but the followed $\mathrm{mRS}$ score was significantly higher among those progressive MIS than those stable MIS ( $2.3 \pm 1.0$ vs $0.1 \pm 0.3$, $p<0.001)$. Conclusion: MIS had a very high prevalence and different clinical types. Stable MIS is a benign stroke, whereas those progressive MIS may have long-term instability or acute and chronic progressive trend. This information is important in prospectively determining outcome of MIS and in patient treatment.
\end{abstract}

\section{Keywords}

Minor Ischemic Stroke, Magnetic Resonance Imaging, Incidence, Clinical Types, Outcome 


\section{Introduction}

With magnetic resonance imaging (MRI) advances, several epidemiological studies have reported that the incidence of minor ischemic stroke (MIS) is up to $55 \%-74 \%$ in metropolitan regions [1] [2], showing MIS is a common ischemic stroke appearing in world regions. In such studies, small stroke or lacunar stroke was usually considered favorable short-term outcome and having had a very low NIHSS score, so this is why it is considered a MIS [3] [4]. In fact, it has been shown that MIS may also result from atheromatous stenosis or occlusion of larger arteries [4] [5] [6], in which the presence of one or more lesions may be associated with a rapidly progressive deficit [7] [8] [9], or even carry a worse prognosis [9] [10]. Thus, MIS has become a common concern public health problem. However, because of MIS's lack of an available clinical classification, it has long been a controversy whether MIS is a benign stroke or not. The aim of present study is to clarify and define the features in clinical types, survival rate, and outcome of MIS in older adults from the villages and towns in northern China.

\section{Methods}

\subsection{Study Population}

From February 2011 to February 2012, a retrospective cohort of new patients was selected for study, all enrolled neurologic outpatients of the tertiary teaching hospital in northern Jiangsu, China. A total of 433 neurologic outpatients, clinically only having had their initial visit and having undergone the MRI studies of the brain, were prospective registered. The sample consisted of males and females, aged 40 years or older, residing in 38 villages or towns and one urban area in Shuyang. The study was approved by the Ethical Committee on Clinical Research of the Shuyang People' hospital, China. All participants gave written informed consent to participate in the study. Clinical investigations were conducted according to the principles expressed in the Declaration of Helsinki.

\subsection{Identified of Patients}

We evaluated those outpatients who had an ischemic stroke based on the International Statistical Classification of Diseases, 10th Revision (ICD-10). In this study, the inclusion criteria of MIS were as follows: 1) first visit indicating minor brain symptoms, with or without minor positive signs of stroke, and measured as $\leq 3$ on the National Institutes of Health Stroke Scale (NIHSS); 2) an MRI displaying visible MIS as a small infarcts within 24 hours of initial presentation, or lacunar lesions or increased brain signal using diffusion weighted imaging (DWI) or fluid-attenuated inversion recovery (FLAIR), with a location in the subcortical white matter, the basal ganglia, or the brain stem. Exclusion criteria were as follows: 1) age $<25$ years; 2) previous history of stroke diagnosed; 3 ) infarct size $>25 \mathrm{~mm}$; or 4 ) an NIHSS score of $>3$ on first visit. 


\subsection{Data Collection}

Information obtained from each patient at baseline included data on gender, age, body mass index (BMI), episode duration, clinical manifestations, and the imaging of brain MRI in initial visit. The following risk factors were obtained from outpatients registered: BMI measured at baseline was categorized as normal $\left(\mathrm{BMI}<25 \mathrm{~kg} / \mathrm{m}^{2}\right)$, overweight $\left(25-30 \mathrm{~kg} / \mathrm{m}^{2}\right)$, or obese $\left(>30 \mathrm{~kg} / \mathrm{m}^{2}\right)$. Hypertension was defined if a patient had a clinical history of hypertension, or had two or more previously documented systolic blood pressures $>160 \mathrm{~mm} \mathrm{Hg}$ or diastolic blood pressures $>90 \mathrm{~mm} \mathrm{Hg}$. Diabetes mellitus was deemed present if the patient gave a history of the condition that was confirmed in the medical records, or there was a random blood glucose concentration of $>11 \mathrm{mmol} / \mathrm{l}$. Dyslipidemia was deemed present if total venous plasma cholesterol level $>6.0 \mathrm{mmol} / \mathrm{L}, \mathrm{LDL}$ fraction $>3.0 \mathrm{mmol} / \mathrm{L}$, triglyceride level $>1.8 \mathrm{mmol} / \mathrm{L}$. Coronary heart disease was defined as a history of acute myocardial infarction or angina pectoris. Atrial fibrillation indicated present when seen on an electrocardiogram during the initial visit or when it was reported in medical records. Cigarette smoking was defined as a last 5 years.

All MR (performed with 1.5-T equipment) studies were reviewed by a neuroradiologist and a neurologist who were blinded to the study. The examiners looked specifically for hyperintense lesions on FLAIR or DWI, measuring maximum diameters when present and attempting to correlate MR findings with clinical status. The number and location of lesions were recorded in detail for each patient.

\subsection{Outcome Evaluation}

A neurology specialist who made the baseline assessments followed patients until 6 months after their index event or the time of death if it occurred before that date. All patients were reassessed during the 6 months follow-up, and were classified as "no symptoms or minor symptoms" (score 0 or 1), "lifestyle restriction" [2] [3], "dependent" [4] [5] according to the stroke-related handicap scale [11], and "death" [6] in our study. The follow-up information was gathered by the same neurology specialist, who conducted inquiries by phone. If the patient was dead or otherwise not assessable, information was obtained from the patient's closest relative or another reliable proxy (follow-up began in Jan 2013 and ended in Jun 2013).

The criteria of a clinical classification for subtypes of MIS were applied that combined clinical data (including the severity and duration of $\mathrm{mRS}$ score during the follow-up) with the results of MRI so that strokes could be classified into several "outcome distinct" groups. In accordance with clinical features and followed mRS score, two types of MIS were delineated: 1) Stable MIS: those MIS with a stable or benign course [3] [4], and with a followed mRS score of 0 - 1;2) Progressive MIS: including two subtypes (a) acute progressive MIS: any symptoms rapidly progress or recurrent new symptoms, and lesions rapidly evolution or extension during the first 7 days after onset; or adds at least 1 point to scores 
by National Institutes of Health Stroke Scale (NIHSS) [8], and with a followed mRS score of 2 - 6 at 6 months follow-up; (b) chronic progressive MIS: symptoms slowly occurring for more than a few months; frequent transient symptoms or new ischemic event tend to progress slowly; or with chronic progressive neurologic decline and normal pressure hydrocephalus [12], or chronic or acute-onchronic white matter ischaemia [13], and with a mRS score of $2-6$ at 6 months follow-up.

\subsection{Related Definition}

The transient symptoms included transient ischemic attack (TIA) and transient neurological attack (TNA). The newly proposed definition of TIA is a transient episode of neurological dysfunction caused by focal brain, spinal cord, or retinal ischemia, without acute infarction [14]. TNA is defined as attacks with temporary $(<24$ hours $)$ neurological symptoms, and these symptoms can be focal, nonfocal, or a mixture of both [15].

The syndromes classified by Fisher, namely pure sensory stroke, pure motor syndromes, ataxic hemiparesis, dysarthria clumsy hand, and sensorimotor stroke, were included in this study. The atypical, nonfocal or mixed symptoms such as headache or/and dizziness which can be attributed to a MIS, were summed as follow.

Headache/migraine is a common symptom and risk factor for cerebrovascular disease [16] [17]. Dizziness/vertigo is also a common cerebrovascular symptom [12] [18], and dizziness or vertigo has an increased risk of developing vascular events [19] [20]. The above dizziness and headache may develop alternately or together. These problems are usually recurring. The duration of symptoms can be from minutes to hours (generally $<24$ hours), although some may be re- current or continuous for a few days or more.

\subsection{Statistical Analysis}

All numeric variables were expressed as the mean \pm SD or median (interquartile range [IQR]). Fisher's Exact test and Kruskal-Wallis test were used to explore the relationship among baseline patient variables. Continuous variables were compared using the $t$ test. Univariate analyses were used to determine risk factors of MIS. Logistic regression analyses for risk factors between stable MIS and progressive MIS were used to calculate Odd ratios (OR), 95\% confidence intervals (CI), and probability values. Kaplan-Meier method and test were used to estimate the differences in survival between groups. Multivariate regression analysis by Cox proportional hazards model was performed for each baseline factor. Data were analyzed using SPSS version 17.0 (SPSS Inc., Chicago, IL, USA), with level of significance set at $P<0.05$.

\section{Results}

Of 433 outpatients, 247 (57\%) patients with MIS were diagnosed according to findings on brain MRI. $90 \%$ of patients with MIS were released at home received 
aspirin treatment. The rest (about 10\%) were admitted to a neurologic ward.

\section{Features of subtype among patients with MIS}

Baseline characteristics and types of MIS on out-patient clinic and during a follow-up are displayed in Table 1. The stable MIS was most frequent (58.3\%). Transient symptoms (TIAs or TNAs) were observed in up to $80 \%$ of stable MIS patients. With acute or subacute onset and relatively stable symptoms, a 8.6-day median (range 1017 days) was recorded for these stable episodes. The stable MIS was solitary in 19 patients and multiple in 125.

Table 1. Clinical characteristics by disease type in patients with MIS*

\begin{tabular}{|c|c|c|c|c|}
\hline \multirow{2}{*}{ Characteristics } & \multirow{2}{*}{$\begin{array}{c}\text { Stable MIS } \\
(\mathrm{N}=144)\end{array}$} & progressive & MIS & \multirow{2}{*}{$P$ Value } \\
\hline & & $\begin{array}{l}\text { AP-MIS } \\
(\mathrm{N}=13)\end{array}$ & $\begin{array}{l}\text { CP-MIS } \\
(\mathrm{N}=90)\end{array}$ & \\
\hline Male gender (\%) & $73(50.7)$ & $9(69.2)$ & $40(44.4)$ & 0.460 \\
\hline Median age, years (range) & $57(40-84)$ & $74(65-83)$ & $61(42-85)$ & 0.001 \\
\hline Residing in villages or towns & $135(93.8)$ & $12(92.3)$ & $85(94.4)$ & 0.948 \\
\hline Residing in county urban & $9(6.2)$ & $1(7.7)$ & $5(5.6)$ & 0.948 \\
\hline Median episode duration, days (range) & $8.6(1017)$ & $2.5(5)$ & $180(2879)$ & 0.001 \\
\hline SBP & $151.3 \pm 20.7$ & $164.9 \pm 23.3$ & $150.6 \pm 22.8$ & 0.139 \\
\hline DBP & $96.3 \pm 10.1$ & $94.8 \pm 10.2$ & $95.4 \pm 13.3$ & 0.713 \\
\hline Blood glucose $(\mathrm{mmol} / \mathrm{l}$, mean $\pm \mathrm{SD})$ & $5.7 \pm 1.6$ & $5.6 \pm 1.5$ & $5.9 \pm 2.3$ & 0.649 \\
\hline Migraine/headache (\%) & $34(23.6)$ & $1(7.7)$ & $21(22.3)$ & 0.336 \\
\hline its TIAs or TNAs (\%) & $30(88.2)$ & $0(0.0)$ & $21(100.0)$ & 0.151 \\
\hline Dizziness/vertigo (\%) & $52(36.1)$ & $5(38.5)$ & $18(20.0)$ & 0.023 \\
\hline its TIAs or TNAs (\%) & $46(88.5)$ & $0(0.0)$ & $18(100.0)$ & 0.007 \\
\hline Headache and dizziness (\%) & $34(23.6)$ & $4(30.8)$ & $31(34.4)$ & 0.194 \\
\hline its TNAs (\%) & $26(76.5)$ & $0(0.0)$ & $28(90.3)$ & 0.009 \\
\hline Pure sensory stroke (\%) & $16(11.1)$ & $2(15.4)$ & $15(16.7)$ & 0.414 \\
\hline its TIAs & $13(81.3)$ & $0(0.0)$ & $10(66.7)$ & 0.429 \\
\hline Pure motor hemiparesis (\%) & $3(2.1)$ & $6(46.1)$ & $1(1.1)$ & 0.001 \\
\hline Gait disorderss (\%) & $8(5.6)$ & $2(15.4)$ & $9(10.0)$ & 0.317 \\
\hline Miscellaneous (\%) & $4(2.8)$ & $0(0.0)$ & $2(2.2)$ & 0.787 \\
\hline Single LI (\%) & $19(15.0)$ & $0(0.0)$ & $0(0.0)$ & 0.001 \\
\hline Chronic lacunes (\%) & $0(0)$ & $0(0)$ & $4(4.4)$ & 0.024 \\
\hline Leukoaraiosis (\%) & $2(0.14)$ & $1(7.7)$ & $13(14.4)$ & 0.001 \\
\hline Normal pressure hydrocephalus (\%) & $0(0)$ & $0(0)$ & $5(5.6)$ & 0.009 \\
\hline Initial median lesions number (range) & $5.5(18)$ & $25(105)$ & $9(80)$ & 0.001 \\
\hline Initial median infarcts volume $\left(\mathrm{cm}^{3}\right.$, range $)$ & $0.3(4.5)$ & $4.1(9)$ & $0.9(8)$ & 0.001 \\
\hline Initial median NIHSS score (range) & $0.1(3)$ & $1(3)$ & $0.2(3)$ & 0.001 \\
\hline Initial median ABCD2 score (range) & $4.5(6)$ & $5.3(6)$ & $4.8(7)$ & 0.014 \\
\hline Followed median mRS score (range) & $0.2(1)$ & $3.0(6)$ & $2(6)$ & 0.001 \\
\hline Mortality (\%) & $0(0)$ & $1(7.7)$ & $1(1.1)$ & 0.074 \\
\hline
\end{tabular}

${ }^{*} \mathrm{MIS}=$ Minor ischemic stroke; AP-MIS = Acute progressive MIS; CP-MIS = Chronic progressive MIS; TIA $=$ Transient ischemic attack; TNA $=$ Transient neurological attack; SBP $=$ Systolic blood pressure; DBP = Diastolic blood pressure; NIHSS = national Institutes of Health Stroke Scale; ABCD2 = Age, blood pressure, clinical features, duration of symptoms, and presence or absence of diabetes; scores range from 0 to 7 , with higher scores indicating greater risk of stroke; $\mathrm{mRS}=$ modified Rankin scale. 
The acute progressive type of MIS (AP-MIS) was the least frequent (5.3\%) and rapid deterioration was fairly typical, running a 2.5-day median course (range 5 days). Such lesions rapidly progressed, in a matter of hours or days, to affect multiple areas supplied by deep perforators, either individually or in aggregate as relatively large infarctions.

Chronic progressive type of MIS (CP-MIS) is the moderate frequency (36.4\%), marked by an unstable clinical course and relatively slow progression (median course of 180 days). With this type of MIS, transient symptoms (TIAs or TNAs ) developed in $85.5 \%$ of those afflicted. On MRI, all instances were multifocal MIS and from one vascular supply slowly involves other sources. Eventually, many regions of the brain were affected, signaling anterior and posterior circulatory compromise.

\section{Features of clinical symptoms in patient with MIS}

There was no difference in gender, SBP, DBP, blood glucose, headache/ migraine, dizziness and headache, pure sensory stroke, gait disorders, miscellaneous, and mortality among stable MIS, AP-MIS, and CP-MIS. Single MIS and dizziness/vertigo were more prevalent in stable MIS than in progressive MIS ( $p$ $<0.05$ ). Older age, pure motor hemiparesis, and high NIHSS score were significantly more prevalent in AP-MIS than in stable MIS and CP-MIS $(p<0.05)$. Longer episode duration, chronic lesion, leukoaraiosis, and normal pressure hydrocephalus were significantly more prevalent in CP-MIS than in stable MIS and AP-MIS $(p<0.05)$. The number of lesions, volume of infarcts, INHSS score, and $\mathrm{ABCD}^{2}$ score were significantly higher in AP and CP-MIS than in stable MIS ( $p$ $<0.05)$ (Table 1).

\section{Risk factors and early prediction in patient with MIS}

Demographics and risk factor profiles in patients with stable MIS and those with progressive MIS were compared (Table 2). The multivariate logistic regression showed that there was no significantly difference in sex, BMI, hypertension, hyperlipidemia, diabetes, heart disease, heavy alcohol drinker, and current smoking between the two groups $(p>0.05)$. Nevertheless, only the age $(\mathrm{OR}=$ $1.059,95 \% \mathrm{CI}=1.026-1.982, p=0.001)$ was a risk factor associated with the stable MIS and progressive MIS (Table 2).

Outcome and survival rate among patients with MIS

The difference was significantly greater when the analysis was confined to followed median mRS score between the stable MIS and the progressive MIS (median 0.2 score versus 2 score, $p=0.001$ ), and the respective mortality rates were $0 \%$ in stable MIS and $1.9 \%$ in progressive MIS (Table 1). Logistic regression analysis of the likelihood of prognosis after adjusted odd ratios (OR) for the baseline and clinical features, only aged (OR, 1.0; 95\% CI, $1.007-1.087, p=$ 0.021 ), episode duration (OR, 1.8; 95\% CI, $1.011-1.024, p=0.000$ ), initial number of MIS (OR, 1.1; 95\% CI, $1.047-1.207, p=0.001$ ), and infarcts volume (OR, 1.8; 95\% CI, $1.253-2.681, p=0.002)$ were independently associated with stable MIS and progressive MIS (Table 3 ).

Survival data were available for our patients with MIS (only two cases of pa- 
Table 2. The baseline risk factor comparison between stable MIS and progressive MIS: OR for each risk factor.

\begin{tabular}{ccccc}
\hline Variable & $\begin{array}{c}\text { Stable MIS } \\
(\mathrm{N}=144)\end{array}$ & $\begin{array}{c}\text { Progressive MIS } \\
(\mathrm{N}=103)\end{array}$ & $\begin{array}{c}\text { OR } \\
(95 \% \text { CI for OR })\end{array}$ & $\begin{array}{c}P \\
\text { Value }\end{array}$ \\
\hline Male gender (\%) & $73(50.7)$ & $49(47.6)$ & $1.4(0.767-2.731)$ & 0.254 \\
Age (y, mean \pm SD) & $58.2 \pm 10.5$ & $63.7 \pm 11.2$ & $1.1(1.025-1.081)$ & 0.001 \\
BMI ( kg/m², mean \pm SD) & $23.9 \pm 2.9$ & $23.9 \pm 3.4$ & $1.0(0.939-1.129)$ & 0.533 \\
Hypertension (\%) & $87(60.4)$ & $59(47.3)$ & $0.7(0.396-1.225)$ & 0.209 \\
Diabetes mellitus (\%) & $9(6.3)$ & $10(9.7)$ & $1.7(0.625-4.709)$ & 0.295 \\
Coronary heart disease (\%) & $5(3.5)$ & $2(1.9)$ & $0.4(0.067-2.034)$ & 0.253 \\
Atrial fibrillation (\%) & $1(0.7)$ & $1(1.0)$ & $1.6(0.089-28.77)$ & 0.749 \\
Hyperlipidemia (\%) & $83(57.6)$ & $58(56.3)$ & $0.8(0.462-1.423)$ & 0.465 \\
Heavy alcohol drinker (\%) & $43(29.9)$ & $17(16.5)$ & $0.5(0.222-1.036)$ & 0.061 \\
Current smoking (\%) & $41(28.5)$ & $20(19.4)$ & $0.6(0.265-1.284)$ & 0.181 \\
\hline
\end{tabular}

MIS = Minor ischemic stroke; $\mathrm{OR}=$ Odd ratio $\mathrm{CI}=$ Confidence intervals; $\mathrm{BMI}=$ Body mass index.

Table 3. Logistic regression analysis for the likelihood of prognosis after adjustment for the baseline and clinical features in stable MIS and progressive MIS.

\begin{tabular}{ccccc}
\hline Variable & $\begin{array}{c}\text { Stable } \\
\text { MIS (mean } \pm \text { SD) }\end{array}$ & $\begin{array}{c}\text { Progressive } \\
\text { MIS (mean } \pm \text { SD) }\end{array}$ & $\begin{array}{c}\text { OR } \\
(95 \% \text { CI for OR) }\end{array}$ & $\begin{array}{c}p \\
\text { Value }\end{array}$ \\
\hline Age (y) & $58.2 \pm 10.5$ & $63.7 \pm 11.2$ & $1.0(1.007-1.087)$ & 0.021 \\
Episode duration (day) & $25.3 \pm 87.0$ & $321.7 \pm 505.0$ & $1.0(1.011-1.024)$ & 0.001 \\
Initial lesions number & $6.3 \pm 4.0$ & $16.4 \pm 18.4$ & $1.1(1.047-1.207)$ & 0.001 \\
Initial infarcts volume $\left(\mathrm{cm}^{3}\right)$ & $0.6 \pm 0.8$ & $2.1 \pm 2.2$ & $1.8(1.253-2.618)$ & 0.002 \\
\hline
\end{tabular}

MIS = Minor ischemic stroke; $\mathrm{OR}=$ Odd ratio; $\mathrm{CI}=$ Confidence intervals

tients lost to follow-up), with a mean follow-up of 180 days. A total of two patients due to the progress of MIS died during follow-up. Survival rate was slightly higher among patients with stable MIS than among those with progressive MIS, but the difference was small (Log rank 2.8; $p=0.094$ ). In Cox proportional hazards analysis, the interaction between the effect of $\mathrm{mRS}$ score and type of MIS on survival was significant ( $\mathrm{OR}=2.6,95 \% \mathrm{CI}=1.049-6.461, p=0.039)$.

\section{Discussion}

With increasing longevity, existing clinical data indicate a 55\% - 74\% incidence of MIS in metropolitan areas [1] [2]. However, in an out-patient setting from the county town and villages in patients over the age of 40 years, our findings show a $57.0 \%$ rate of MIS and with different types in clinical.

Some MIS with a stable course and a good prognosis have been reported [3] [4]. Within our out-patient clinic setting, the stable MIS occurred with greatest frequency, accounting for $58.3 \%$ of patients. Nevertheless, we found that those with stable MIS were younger than those AP-MIS and CP-MIS, possibly due to the presence of a potentially small-artery disease [4] [12]. 
Progressive MIS had been proposed by Bang [7] [8] [9] and Fisher [12]. On our out-patient clinic, 5.3\% of AP-MIS and $36.4 \%$ of CP-MIS were diagnosed. The clinical features of CP-MIS are multiple lesions, recurrent transient symptoms, longer duration (median course of 180 days). It is noted that those patients with recurrent transient symptoms for more than a 180-days median should be estimated to its having a long duration of TIAs or TNAs. The recurrent TIA symptoms or ischemic event is highest in the first month and year among those with a long history of onset [21], probably reflecting the vascular stenosis of the parent artery [6], or presence of active, unstable atherosclerotic plapue [21].

Acute progressive type of MIS occurred in $4.5 \%-6.8 \%$ of the patients with MIS [7] [9], even high to $15.2 \%$ [22], and was often due to stenosis or occlusion of the middle cerebral artery and proximal segments of its deep perforating branches [6] [23] [24]. However, patients experiencing rapid deterioration of MIS, especially sudden and progressive symptoms (not limited to motor deficits), and needing emergency care should rightly be included here. The present study showed that, in older individuals, MIS were more likely to show rapid, focal expansion (a 2.5-day median course).

Previous studied has been shown that the survival rate in MIS hospitalized was $97 \%$ at 1 year [25]. In the present study, the survival rate of stable MIS is $100 \%$, while the survival rate of progressive MIS is $98.1 \%$. This suggests that the survival rate between patients with stable MIS and progressive MIS is small different. However, we have confirmed that overall survival rate in patients with MIS in the out-patient clinic during the follow-up period of 6 months is favorable (99.2\%). Our view is consistent with more studies reported survival of MIS [3] [10]. Although there are one to two reports described poor survival [26] [27], we still believe that the survival status in patients with MIS have a favorable trends.

But then, this does not mean that the survival quality of MIS patients will be long-term stability and excellent health. Moreover, we have shown that the median $\mathrm{mRS}$ score in progressive MIS in the follow-up 6 months was significantly higher than those stable MIS. This finding suggests that progressive MIS may have long-term instability or acute and chronic progressive trend. Some studies have shown that the trends of prognosis for MIS related to the burden of atherosclerosis [16] [27]. In fact, in addition to the presence of vascular risk factors can accelerate the progress of atherosclerosis, the aging is also its important mechanism [28]. This has been confirmed by our present study.

Some limitations of our study are conceded. Because the mRS gap for each of the subgroups were very narrow, misclassification between stable MIS and progressive MIS may be presented by the data on phone. In fact, these mRS scores were markedly differences between the "no symptoms or minor symptoms" (score 0 or 1) and "lifestyle restriction" ( 2 - 3). Therefore, we believe that at most a very small case would have misclassified in the inquiries. In addition, the absence of a repeated MRI or vascular imaging data from some patients may be affected the predictors for patients with progressive MIS as well as recurrent events. 


\section{Conclusion}

MIS had a very high prevalence and different clinical types. Stable MIS is a benign stroke, whereas those progressive MIS may have long-term instability or acute and chronic progressive trend. This information is important in determining outcome of MIS and in patient treatment.

\section{Acknowledgements}

This work is supported by a grant from the Medical Research Council, affiliated Shuyang People's Hospital, XuZhou Medical University, China.

\section{Sources of Funding}

Clinical Key Specialty Construction Project of Jiangsu Provence (20120013).

\section{References}

[1] Wang, L, Chao, Y, Zhao, X, Liu, L, Wang, C, Wang, D.Z., et al. (2013) Factors Associated with Delayed Presentation in Patients with TIA and Minor Stroke in China: Analysis of Data from the China National Stroke Registry (CNSR). Neurological Research, 35, 517-521. https://doi.org/10.1179/1743132813Y.0000000204

[2] Chandratheva, A., Lasserson, D.S., Geraghty, O.C. and Rothwell, P.M. (2010) Oxford Vascular Study. Population-Based Study of Behavior Immediately after Transient Ischemic Attack and Minor Stroke in 1000 Consecutive Patients: Lessons for Public Education. Stroke, 41, 1108-1114. https://doi.org/10.1161/STROKEAHA.109.576611

[3] Fischer, U., Baumgartner, A., Arnold, M., Nedeltchev, K., Gralla, J., De Marchis, G.M., et al. (2010) What Is a Minor Stroke? Stroke, 41, 661-666. https://doi.org/10.1161/STROKEAHA.109.572883

[4] Bang, O.Y., Joo, S.Y., Lee, P.H., Joo, U.S., Lee, J.H., Joo, I.S., et al. (2004) The Course of Patients with Lacunar Infarcts and a Parent Arterial Lesion: Similarities to Large Artery vs Small Artery Disease. Archives of Neurology, 61, 514-519. https://doi.org/10.1001/archneur.61.4.514

[5] Norrving, B. and Cronqvist, S. (1989) Clinical and Radiologic Features of Lacunar versus Nonlacunar Minor Stroke. Stroke, 20, 59-64. https://doi.org/10.1161/01.STR.20.1.59

[6] Bang, O.Y., Heo, J.H., Kim, J.Y., Park, J.H. and Huh, K. (2002) Middle Cerebral Artery Stenosis Is a Major Clinical Determinant in Striatocapsular Small, Deep Infarction. Archives of Neurology, 59, 259-263. https://doi.org/10.1001/archneur.59.2.259

[7] Ferrari, J., Knoflach, M., Kiechl, S., Willeit, J., Schnabl, S., Seyfang, L., et al. (2010) Early Clinical Worsening in Patients with TIA or Minor Stroke: The Austrian Stroke Unit Registry. Neurology, 74, 136-141. https://doi.org/10.1212/WNL.0b013e3181c9188b

[8] Yamamoto, Y., Ohara, T., Hamanaka, M., Hosomi, A., Tamura, A., Akiguchi, I., et al. (2010) Predictive Factors for Pregressive Motor Deficits in Penetrating Artery Infarctions in Two Different Arterial Territories. Journal of the Neurological Sciences, 288, 170-174. https://doi.org/10.1016/j.jns.2009.08.065

[9] Ogata, T., Yasaka, M., Wakugawa, Y., Ibayashi, S. and Okada, Y. (2009) Predisposing Factors for Acute Deterioration of Minor Ischemic Stroke. Journal of the Neurological Sciences, 287, 147-150. https://doi.org/10.1016/j.jns.2009.08.006 
[10] van Wijk, I., Lindeman, E., Kappelle, L.J., van Gijn, J., Koudstaal, P.J., Gorter, J.W., et al. (2006) Functional Status and Use of Healthcare Facilities in Long-Term Survivors of Transient Ischaemic Attack or Minor Ischaemic Stroke. Journal of Neurology, Neurosurgery \& Psychiatry, 77, 1238-1243. https://doi.org/10.1136/jnnp.2006.089391

[11] Van Swieten, J.C., Koudstaal, P.J., Visser, M.C., Schouten, H.J.A. and van Gijn, J. (1998) Interobserver Agreement for the Assessment of Handicap in Stroke Patients. Stroke, 19, 604-607. https://doi.org/10.1161/01.STR.19.5.604

[12] Fisher, C.M. (1982) Lacunar Strokes and Infarcts: A Review. Neurology, 32, 871876. https://doi.org/10.1212/WNL.32.8.871

[13] Mathers, S.E., Chambers, B.R., Merory, J.R. and Alexander, I. (1987) Subcortical Arteriosclerotic Encephalopathy: Binswanger's Disease. Clinical and Experimental Neurology, 23, 67-70.

[14] Easton, J.D., Saver, J.L., Albers, G.W., et al. (2009) Definition and Evaluation of Transient Ischemic Attack: A Scientific Statement for Healthcare Professionals from the American Heart Association/American Stroke Association. Stroke, 40, 22762293. https://doi.org/10.1161/STROKEAHA.108.192218

[15] Bos, M.J., van Rijn, M.J., Witteman, J.C., Hofman, A., Koudstaal, P.J. and Breteler, M.M. (2007) Incidence and Prognosis of Transient Neurological Attacks. JAMA, 298, 2877-2885. https://doi.org/10.1001/jama.298.24.2877

[16] Bousser, M.G. and Welch, K.M. (2005) Relation between Migraine and Stroke. Lancet Neurology, 4, 533-542. https://doi.org/10.1016/S1474-4422(05)70164-2

[17] Stang, P.E., Carson, A.P., Rose, K.M., Mo, J., Ephross, S.A., Shahar, E., et al. (2005) Headache, Cerebrovascular Symptoms, and Stroke: The Atherosclerosis Risk in Communities Study. Neurology, 64, 1573-1577. https://doi.org/10.1212/01.WNL.0000158326.31368.04

[18] Cloutier, J.F. and Saliba, I. (2008) Isolated Vertigo and Dizziness of Vascular Origin. Journal of Otolaryngology_Head \& Neck Surgery, 37, 331-339.

[19] Okroglic, S., Widmann, C.N., Urbach, H., Scheltens, P. and Heneka, M.T. (2013) Clinical Symptoms and Risk Factors in Cerebral Microangiopathy Patients. PLoS ONE, 8, e53455. https://doi.org/10.1371/journal.pone.0053455

[20] Yamasoba, T., Kikuchi, S., Higo, R., Kaga, K., O’uchi, T. and Tokumaru, A. (1993) Ischemic Brain Lesions in Aged Patients with Dizziness. Archives of Otolaryngology-Head \& Neck Surgery, 119, 1346-1350. https://doi.org/10.1001/archotol.1993.01880240084010

[21] Hankey, G.J. (2003) Long-Term Outcome after Ischaemic Stroke/Transient Ischaemic Attack. Cerebrovascular Diseases, 16, 14-19. https://doi.org/10.1159/000069936

[22] Ju,Y., Zhao, X.Q., Wang, C.X., Wang, Y.L., Liu, G.F. and Wang, Y.J. (2013) Neurological Deterioration in the Acute Phase of Minor Ischemic Stroke Is an Independent Predictor of Poor Outcomes at 1 Year: Results from the China National Stroke Registry (CNSR). Chinese Medical Journal, 126, 3411-3416.

[23] Lee, D.K., Kim, J.S., Kwon, S.U., Yoo, S.H. and Kang, D.W. (2005) Lesion Patterns and Stroke Mechanism in Atherosclerotic Middle Cerebral Artery Disease: Early Diffusion-Weighted Imaging Study. Stroke, 36, 2583-2588. https://doi.org/10.1161/01.STR.0000189999.19948.14

[24] Kim, J.T., Kim, H.J., Yoo, S.H., Park, M.S., Kwon, S.U., Cho, K.H., et al. (2010)MRI Findings May Predictearly Neurologic Deterioration in Acute Minor Stroke or Transient Ischemic Attack Due to Intracranial Atherosclerosis. European Neurology, 64, 95-100. https://doi.org/10.1159/000315138 
[25] Staals, J., van Raak, L., Hilton, A. and Lodder, J. (2008) Differences in Long-Term Survival in Two Lacunar Stroke Types: A 15-Year Follow-Up Study in 782 Cerebral Infarct Patients. Cerebrovascular Diseases, 25, 26-31. https://doi.org/10.1159/000111496

[26] Micheli, S., Agnelli, G., Palmerini, F., Caso, V., Venti, M., Alberti, A., et al. (2008) Need for Extensive Diagnostic Work-Up for Patients with Lacunar Stroke. Journal of Neurology, 255, 637-642. https://doi.org/10.1007/s00415-008-0762-5

[27] Laurent, S., Boutouyrie, P. and Lacolley, P. (2005) Structural and Genetic Bases of Arterial Stiffness. Hypertension, 45, 1050-1055. https://doi.org/10.1161/01.HYP.0000164580.39991.3d

[28] Farkas, E. and Luiten, P.G. (2001) Small-Vessel Disease Increases with Age and Is Accelerated by Vascular Risk Factors, Most Notably, Hypertension and Diabetes. Progress in Neurobiology, 64, 575-611. https://doi.org/10.1016/S0301-0082(00)00068-X

Submit or recommend next manuscript to SCIRP and we will provide best service for you:

Accepting pre-submission inquiries through Email, Facebook, LinkedIn, Twitter, etc. A wide selection of journals (inclusive of 9 subjects, more than 200 journals) Providing 24-hour high-quality service User-friendly online submission system Fair and swift peer-review system Efficient typesetting and proofreading procedure Display of the result of downloads and visits, as well as the number of cited articles Maximum dissemination of your research work

Submit your manuscript at: http://papersubmission.scirp.org/ Or contactwjns@scirp.org 